\title{
El Proyecto de Innovación SAME: el fomento de la Responsabilidad Social Universitaria en el alumnado de la Universidad de Valencia.
}

\author{
Ana Ancheta Arrabal ${ }^{\mathrm{a}}$, Cristina Pulido Montes ${ }^{\mathrm{b}}, \mathrm{M}^{\mathrm{a}}$ Esther Alventosa Bleda ${ }^{\mathrm{c}}$ y Borja \\ Mateu Luján ${ }^{\text {d }}$ \\ aUniversitat de València, (Ana.Ancheta@uv.es), bUniversitat de València (Cristina.Pulido@uv.es) y \\ cUniversitat de València (Maria.E.Alventosa@uv.es) ${ }^{d}$ Universitat de València (Borja.Mateu@uv.es)
}

\begin{abstract}
Nowadays, the right to quality education remains an aim within the international agendas. In the mission to defense this right, the Global Campaign for Education arises, as an initiative of civil and political awareness for the entitlement. Through the Educational Innovation project of the Global Action Week for Education (GAWE), which is included in this international campaign, from the Faculty of Philosophy and Education Sciences of the University of Valencia and in collaboration with the Coordination of the campaign in the Comunidad Valenciana we intend to give visibility to the fight for the right to quality education. Among the activities developed to achieve this goal, workshops are offered in the different subjects of the degrees taught in the faculty. All these actions are focused on the culmination of the main act of the campaign that takes place in the GAWE, where the educational community makes and exhibits the produced work and a manifiesto, to give visibility to said action and present it in society. The final result of all this is the participation of the students and the development of competences based on the aim of Sustainable Campus of the Social Responsibility of the University related to the partnerships with institutions and sustainability.
\end{abstract}

Keywords: GAWE, Innovation Project, Community Action, Competences, University Social Responsibility.

\section{Resumen}

El Derecho a la Educación de calidad en la actualidad, sigue siendo un objetivo en las agendas internacionales. En la misión de su defensa surge la Campaña de Acción Mundial por la Educación, como una iniciativa de concienciación civil y política por este derecho. Mediante el proyecto de Innovación Educativa de la Semana de Acción Mundial para la Educación (SAME) inmerso en dicha campaña internacional, desde la Facultad de Filosofía y Ciencias de la Educación de la 
Universidad de Valencia y en colaboración con la Coordinadora de la campaña en la Comunidad Valenciana pretendemos dar visibilidad a la lucha por el Derecho a la Educación de calidad. Entre las actividades desarrolladas para conseguir este objetivo se plantean talleres en diferentes asignaturas de las titulaciones impartidas en la facultad. Todas estas acciones están enfocadas a la culminación del acto principal de la campaña que se realiza en la SAME, donde la comunidad educativa realiza y expone el trabajo realizado y un manifiesto, para dar visibilidad a dicha acción y presentarla en sociedad. El resultado final de todo ello, es la participación del alumnado y el desarrollo de competencias basadas en el objetivo de Campus Sostenible de la Responsabilidad Social de la Universidad relacionado con las alianzas con instituciones y la sostenibilidad.

Palabras clave: SAME, Proyecto Innovación, Acción Comunitaria, Competencias, Responsabilidad Social Universitaria.

\section{Introducción}

La Semana de Acción Mundial por la Educación (SAME, en adelante) es una iniciativa de la Campaña Mundial por la Educación (CME, en adelante) como coalición internacional formada por organizaciones no gubernamentales (ONG), sindicatos del entorno educativo, centros escolares y movimientos sociales de muy diverso signo, que se moviliza para reclamar el cumplimiento íntegro de los compromisos internacionales firmados por los Estados para garantizar el acceso a una educación de calidad para todas las personas del mundo.

La CME también constituye el movimiento de la sociedad civil que trabaja para poner fin a la crisis mundial en la educación. Su misión principal es asegurar que los Estados actúen para hacer realidad el derecho de todos y cada uno de los seres humanos a disfrutar de una educación pública, gratuita y de calidad, para lo que trabaja en alrededor de 124 países (CME, 2018). El objetivo de la CME ha venido siendo velar por el cumplimiento de los compromisos adquiridos por estos países en agendas como la Educación para Todos (Dakar, 2000) y los Objetivos de Desarrollo del Milenio (ODM) del año $2000 \mathrm{y}$, aunque los avances en materia educativa han sido notables - por ejemplo, el número de niños y niñas sin escolarizar en la educación primaria se ha reducido en más de un $40 \%$ desde 1999 - aún queda mucho camino por recorrer por hacer realidad estos fines por parte de la comunidad internacional en materia educativa. En el año 2015 se cumplió el plazo tanto de los objetivos de la iniciativa para hacer efectivo el Derecho a la Educación para todas las personas. En este contexto, en el año 2015 los Gobiernos renovaron su 
compromiso con la educación, marcando la ruta a seguir en la nueva Agenda 2030 de Educación, que incluye la Declaración de Incheon (Corea, 2015) y los Objetivos de Desarrollo Sostenible (ODS). Entre ellos, el ODS número cuatro se centra en "garantizar una educación inclusiva, equitativa y de calidad y promover oportunidades de aprendizaje durante toda la vida para todos y todas antes de 2030" (UNESCO, 2016, p. 20).

En este nuevo escenario, la CME continúa su labor anterior mediante la continuación de iniciativas como la SAME como una de las principales actividades de sensibilización y movilización social en una escala mundial que la UNESCO lidera desde hace años. Este organismo internacional participa mediante la movilización de sus redes y asociados, la recopilación y el intercambio de políticas y prácticas, así como la realización de actividades en el mundo entero a través de sus oficinas internacionales. En esta semana conmemorativa y de acción global se recuerda que los esfuerzos y las iniciativas mundiales en pro de la Educación para Todos deben doblarse a fin de velar por el cumplimiento del Derecho a la Educación y exhortar

a todos los gobiernos y los asociados en las tareas del desarrollo, a todos los docentes, padres y proveedores del sector privado, a que eliminen las barreras que impiden el acceso a la enseñanza o que perduran dentro de los sistemas educativos, a fin de hacer realidad la participación plena y equitativa en la vida social de todas las personas (Bokova, 2014, p. 2).

Internacionalmente, desde el año 2001 la Campaña Mundial por la Educación celebra esta Semana de Acción Mundial por la Educación, normalmente en abril, donde los más de 124 países que integran esta campaña trabajan por garantizar el Derecho a la Educación y promover la movilización ciudadana para llamar la atención de la comunidad educativa, de los medios de comunicación, de la sociedad en general y, especialmente, de los representantes políticos sobre la necesidad de hacer real y efectivo el derecho a una educación de calidad para todos y todas en el mundo. En España, la Semana de Acción Mundial forma parte de la campaña anual que se celebra en el mundo entero y es organizada por la Campaña Mundial por la Educación con el objetivo sensibilizar a la opinión sobre la importancia de una Educación para Todos.

Concretamente desde esta experiencia, la SAME supone una semana de movilización ciudadana a la que la Facultad de Filosofía y Ciencias de la Educación de la Universidad de Valencia (UV) se ha venido sumando desde el curso 2013/14 impulsando dicha semana como una acción transversal en diferentes titulaciones de la UV (Pedagogía, Educación Social y Magisterio), apoyada en la implicación del alumnado y, especialmente, del profesorado ya colaborador de la CME, en algunos casos, en ediciones y celebraciones de la misma precedentes. En la Universidad de Valencia, durante los últimos años, se desarrolla dentro de un proyecto de innovación 
que promueve la interrelación entre esta y distintas organizaciones e instituciones de la sociedad valenciana. De este modo, el Proyecto de innovación SAME se consolida atendiendo a la LEY 2/2003 (DOCV, 2003) que recoge en su preámbulo que la Ley Orgánica 6/2001 de Universidades pretende establecer relaciones y vinculaciones recíprocas entre universidad y sociedad. Asimismo, la SAME favorece la participación del alumnado en las diferentes actividades que se realizan, vinculándose a las enseñanzas impartidas en el Grado, como se refleja en los fines del Consejo Social del artículo 2 de la citada LEY 2/2003.

El caso de la SAME es un claro ejemplo de proyecto de innovación educativa basado en el trabajo en equipo multidisciplinar, interdisciplinar e intergeneracional. El hecho de hacer confluir alumnado universitario y no universitario, profesorado universitario y diferentes agentes sociales del resto de la comunidad educativa, en actividades donde se trabajan valores y conceptos que favorezcan la adquisición de herramientas para el desarrollo social sostenible y en pro del Derecho a la Educación de todos y todas, es una manera de potenciar el sentimiento de que todas y todos formamos parte de la Comunidad Educativa. Además, como se explicará en la metodología, es un proyecto de Acción Comunitaria, ya que aglutina diversos colectivos y personas físicas, y está dinamizado por una coordinadora encargada de hacer de puente entre la campaña internacional y la Universidad de Valencia. En esta acción, como se ha dicho, se generan sinergias (procesos de vinculación y apoyo mutuo) que activan la voluntad de las personas participantes a protagonizar la mejora de su realidad (Gomà 2008). En este caso concreto, como la definen Llena y Úcar (2006) se trataría de una acción comunitaria del tipo 2, es decir, con un interés colectivo y unos objetivos predeterminados como es el acceso a la educación de calidad para todas las personas. Se trata de una acción estratégica e ideológica enmarcada en un sistema de valores, es decir, una intervención en la comunidad de aspecto socioeducativo.

A partir de este proyecto de innovación educativa se trabaja con el alumnado para desarrollar una institución universitaria democrática, sostenible y cooperativa. A la vez, que dotar de sentido crítico a las iniciativas de la institución universitaria, como principal agente de movilización social, tal y como en las Jornadas de Diálogos Universidad y Desarrollo Sostenible celebradas en la Agencia Española de Cooperación Internacional para el Desarrollo, se pondría de relieve que el espacio universitario es privilegiado para formar a las personas en todas sus dimensiones a través de diversas estrategias, entre ellas los proyectos de innovación. En este sentido, la Universidad de Valencia, mediante la aprobación del Plan Estratégico 2016-2019, persigue una serie de objetivos concretos en lo que respecta a la Responsabilidad Social Universitaria y que se fomentan a través del proyecto de innovación SAME, desatacamos especialmente el objetivo: 
- RsC02T. Fomentar los valores ligados al desarrollo humano como son la solidaridad, la cooperación, el progreso, la sostenibilidad, la excelencia y la igualdad mediante el desarrollo de su plan.

La Responsabilidad Social Universitaria según Martínez-Usarralde, Lloret-Català y Mas-Gil (2017) permite que:

se consigan beneficios a corto y largo plazo como son la consecución de una coherencia institucional integradora, una pertinencia y permeabilidad social (apertura), una dinámica hacia la innovación inteligente y transparente y una racionalización de la gestión universitaria que mejora la creación de valor social, a través, por ejemplo, de proyectos de desarrollo de la comunidad. (Martínez-Usarralde, Lloret-Català y Mas-Gil, 2017, p. 6).

A la vez, Martínez-Usarralde, Lloret-Català y Mas-Gil (2017) indican que los agentes que forman parte activa del fomento y trabajo de la Responsabilidad Social Universitaria en un nivel interno son el profesorado, los investigadores, el alumnado y el personal administrativo. En el nivel externo serían las empresas, administraciones y organizaciones no gubernamentales o sin ánimo de lucro.

De este modo, el proyecto de la SAME tal y como es descrito cumple con las características de la Responsabilidad Social Universitaria, ya que lo que pretende es concienciar al alumnado y a la comunidad educativa de una realidad que parte de la violación de un derecho que debe ser exigido por su carácter universal y democrático y que a través de la función transversal del proyecto es trabajado de manera colaborativa, en la que el alumnado es parte activa del mismo. La participación de los agentes, tanto internos como externos, comprende a todos los descritos como elementos fundamentales de la Responsabilidad Social Universitaria. La realización de los talleres previos de difusión de la campaña en las aulas y las comisiones de coordinación del proyecto son un conglomerado de todos los agentes descritos. Más adelante, iremos detallando los talleres, los agentes que participan y el carácter de las comisiones que dan sentido al fomento de la Responsabilidad Social Universitaria promocionado por el proyecto SAME en la Facultades de Filosofía y Ciencias de la Educación y la Facultad de Magisterio de la Universidad de Valencia.

\section{Objetivos}

El proyecto de Semana de Acción Mundial por la Educación, una posibilidad para desarrollar la corresponsabilidad social y educativa del alumnado y profesorado universitario se enmarca en el Programa C de Innovación educativa de la UV centrado en "Grupos estables y redes de innovación continua" y que está orientado a la constitución de grupos y redes de innovación a través de la colaboración con profesorado de otras universidades, e incluso de otros niveles educativos. Se trata, 
también, de consolidar las redes iniciadas en las convocatorias de los cursos 2015/16 y 2016/17, y profundizar en el establecimiento de otras nuevas, fruto de la experiencia acumulada con la participación en proyectos de innovación de anualidades anteriores. Desde el curso 2003/2004 la Universidad de Valencia ha realizado un importante esfuerzo institucional para impulsar la innovación educativa en los estudios universitarios y de esta manera colaborar en la mejora de la docencia en el contexto de la construcción del Espacio Europeo de Educación Superior (EEES). Durante los cursos anteriores al 2015/16, las sucesivas convocatorias han tratado de vincular las actuaciones de innovación educativa con el resto de programas estratégicos relacionados con la convergencia europea y la calidad docente de las nuevas titulaciones, sobre todo en cuanto a la utilización y exploración de las potencialidades de las nuevas tecnologías (UV, 2017). Este programa se engarza de manera sinérgica con algunas de las líneas de actuación estratégica que, sin negar la posibilidad de trabajar en otras temáticas, tienen un tratamiento preferente, toda vez que suponen aspectos especialmente vinculados a la mejora de la calidad de la docencia universitaria en la UV (UV, 2017):

- Uso de las TICs y la elaboración de materiales en abierto;

- Fomento de la participación de los y las estudiantes en la mejora de los procesos de enseñanza-aprendizaje

- Mejora de los planes de estudios, con la vista puesta en la acreditación y la obtención de sellos de calidad

- Puesta en valor y transferencia del conocimiento e investigación en materia de innovación educativa.

Aparte de la defensa del Derecho a la Educación para todos y todas, objetivo principal de la SAME, en las actividades desarrolladas se trabaja de manera práctica y teórica contenidos de la Agenda 2030 como, por ejemplo, la meta 4.7 que concretamente se centra en:

De aquí a 2030, asegurar que todos los alumnos adquieran los conocimientos teóricos y prácticos necesarios para promover el desarrollo sostenible, entre otras cosas mediante la educación para el desarrollo sostenible y los estilos de vida sostenibles, los derechos humanos, la igualdad de género, la promoción de una cultura de paz y no violencia, la ciudadanía mundial y la valoración de la diversidad cultural y la contribución de la cultura al desarrollo sostenible (punto 4.7 del Objetivo 4 de la Agenda 2030 de la UNESCO (Declaración Incheon, 2016, p. 21).

Revisando estudios como el de Barrón, Navarrete y Ferrer-Balas (2010), afirman que la formación de profesionales críticos con la manera de proceder respecto al desarrollo sostenible de nuestra sociedad actual es crucial. Ya entonces se intuía que los objetivos de la Agenda 21 no serían fáciles de conseguir, lo que lamentablemente se corroboró posteriormente. Acyalmente la UNESCO plantea nuevos retos, sin 
dejar de insistir en los anteriores, ya que se reafirma en la necesidad de la educación para el desarrollo y los estilos de vida sostenibles.

La acción desarrollada a través del proyecto de innovación de la SAME es intrínsecamente educativa por cuanto constituye un proceso de enseñanzaaprendizaje continuo, transformador y constantemente que es retroalimentado por todas y cada una de las personas implicadas, desde el momento en que se pone en marcha el proyecto, en torno a la consigna sobre la que trabajar pedagógicamente cada año, y que repercute de forma indiscutible en lo que denominamos como el proceso de "Educación a lo largo de la vida" (Life Long Learning) en el que creemos y participamos. El profesorado involucrado, docente en cuatro grados diferentes, entendemos que el proceso de elaboración de las diferentes acciones y actividades de aprendizaje que se van a ir diseñando en torno a la campaña (CME) y que se abocarán a la celebración de un acto de celebración del día de la campaña (SAME) tienen un carácter aplicado, dado que el alumnado que se involucre va a trabajar contenidos, estrategias, habilidades, capacidades y actitudes de carácter académico $\mathrm{y}$, sobre todo, profesionalizador y humanizador. Todo ello redunda en una revisión y certificación de las competencias que se ponen en jaque a lo largo y ancho de la articulación del mismo, donde los objetivos definidos para este proyecto de innovación educativa son los siguientes:

- Impulsar la Responsabilidad Social Universitaria a través del proyecto de innovación SAME.

- Explorar y aprender de la riqueza y oportunidades didáctico-metodológicas que ofrece la Campaña Mundial por la Educación (CME) y la Semana de Acción Mundial por la Educación (SAME).

- Cooperar con las ONGs, las asociaciones involucradas y los centros educativos, incidiendo en la función de responsabilidad social universitaria, fomentando las redes de participación con la sociedad civil.

- Impulsar la creatividad, el carácter propositivo y reactivo, así como los valores relacionados con el compromiso, la participación y la solidaridad bien entendida. Visualizando, de este modo, la realidad del Sur y profundizando los lazos de colaboración.

- Contribuir a la formación integral y de calidad del profesorado y alumnado universitario.

- Profundizar en la riqueza que aporta el trabajo colaborativo y cooperativo entre las diferentes personas y organismos que participan en la SAME.

- Utilizar TICs y redes sociales como medio para de difusión y herramienta de trabajo durante todo el proceso de la acción socio-pedagógica.

Para este trabajo que se presenta en concreto, dichos objetivos son los de la propia innovación, en la medida que el proyecto en sí mismo la persigue como fin. 


\section{Desarrollo de la innovación}

La metodología para llevar a cabo el desarrollo de la innovación parte de estrategias educativas que fomentan la participación, el diálogo, la negociación, la reflexión, la crítica y la evaluación. Esta metodología se viene gestionando a través de comisiones coordinadas por varias personas para facilitar el trabajo colaborativo entre la Coalición Española de la SAME y el profesorado y alumnado de la Universidad. De hecho, es una metodología relacionada con la acción comunitaria, ya que se trata de la acción desarrollada por un colectivo humano (parte de la comunidad), que en este caso se hablaría de la Comunidad Educativa, compartiendo el espacio de la Universidad de Valencia y con conciencia de pertenencia a dicha comunidad, desde la detección de una necesidad y avanzando, año tras año para encontrar una solución conjunta.

Además, cuenta con los principales elementos a tener en cuenta en una acción comunitaria, según Ricard Gomà (2008), relacionados con dinámicas participativas y de construcción de ciudadanía como:

- Autonomía y responsabilidad. Ya que existe una Coordinadora encargada de convocar y dinamizar el proceso, favoreciendo el trabajo conjunto y la construcción de soluciones.

- Confianza y respeto. Existe un vínculo entre las personas que participan y las que lo llevan adelante, que fomenta la confianza y reciprocidad, lo que favorece dinámicas de corresponsabilidad.

- Deliberación y transparencia. Se realizan reuniones informativas y de participación donde poder opinar y proponer aquello que se considere necesario. También se informa del presupuesto disponible para trabajar.

- Conflicto e innovación. Todas las propuestas son escuchadas con respeto y el diálogo es la principal herramienta para resolver conflictos, dejando aflorar la creatividad y la innovación social.

- Complejidad y articulación de redes. Como ya se ha explicado, existe una gran confluencia de diferentes agentes y la articulación de redes basada en interdependencias.

- Dinamismo y aprendizaje. Se trabaja de manera planificada y gestionada, aunque flexible, para crear dinamismo. Se revisan los procesos y contenidos para adaptarse a las circunstancias o la demanda social. Un claro ejemplo es la ampliación progresiva de actividades realizadas desde las redes sociales (TIC), para llegar a las personas más jóvenes y expandir la difusión del evento de manera óptima.

- Proximidad y dinámicas sostenibles. Teniendo en cuenta el tiempo que el proyecto lleva realizándose, es de suponer que se adapta a los recursos de los que dispone, ya sean materiales o humanos. 
Para ello, la metodología de trabajo viene siendo consensuada por el equipo que solicita y el que participa en este proyecto cada curso académico, aprovechando la experiencia en la participación de la organización de la SAME en convocatorias anteriores. El salto cualitativo de este proyecto de innovación parte de la intención de ofrecer la oportunidad a la totalidad de los agentes de la comunidad educativa de participar con su propia voz en los procesos de elaboración de la propuesta y no sólo como receptores finales. Por todo ello, se centra en erigirse en torno a la consolidación de comisiones de trabajo lideradas por 2 o 3 profesores/as junto con el alumnado participante en las mismas:

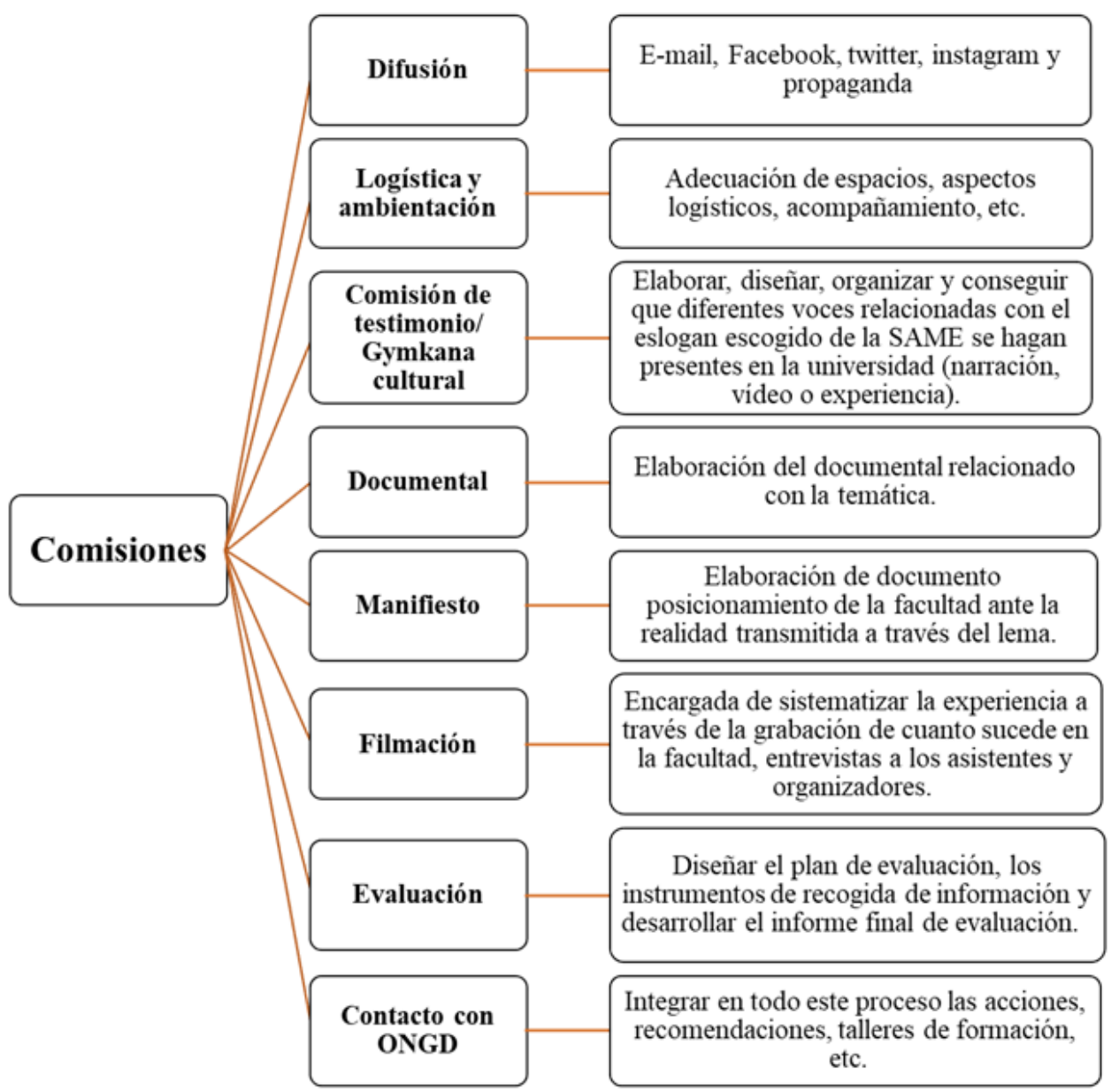

Fig. 1 Comisiones del Proyecto de innovación educativa SAME

Las Comisiones establecidas para este proyecto varían cada año en función de las necesidades, aunque las más representativas son descritas en la Fig.1. Todo este funcionamiento contribuye a que cada persona participante en el proyecto se sienta parte del mismo y adquiera el compromiso y la corresponsabilidad de llevarlo a cabo, así como la satisfacción de colaborar en el resultado final. 


\section{Resultados}

El profesorado y el alumnado de este proyecto transversal, interdepartamental e interdisciplinar están adscritos a cuatro grados relacionados con educación: Educación Social, Pedagogía, Filosofía y Magisterio. Su desarrollo e implementación ha demostrado las posibilidades pedagógicas que se abren al aprendizaje cooperativo interdisciplinar, ejercitándose en torno al proyecto de innovación generado a partir de los actos de la SAME, siendo los resultados sumamente satisfactorios tanto para el profesorado involucrado como para el alumnado, que lo ha venido reflejando en las encuestas llevadas cabo durante las ediciones anteriores de este proyecto de innovación. Consecuentemente, en aras de seguir incidiendo en esta metodología transversal, el profesorado participante se ha constituido como Grupo estable y red de innovación continua dentro del mencionado programa de innovación de la UV, posibilitando la estabilidad de la experiencia y permanencia de los vínculos de colaboración entre diversos agentes de la comunidad universitaria como del resto de la comunidad educativa valenciana. A través de los protagonistas, profesorado y alumnado de dichos grados, pero también de los expertos invitados, de las ONGD que lideran la SAME en la Comunidad Valenciana y de los colegios intervinientes, se hace patente que el éxito de un proyecto así entendido se genera desde el compromiso y del trabajo bien hecho de todos y cada uno de sus participantes. El resultado pone de manifiesto en cada acto de celebración y culminación de la SAME que el alumnado implicado está capacitado para abordar, desde su profesión pero también desde su responsabilidad como miembro de una ciudanía global, su cuota de acción profesionalizadora y ciudadana.

A lo largo de las ediciones de este proyecto realizadas desde el curso 2013/2014 hasta el actual, los resultados se han analizado en relación a los distintos modos de producción y de participación que el profesorado y el alumnado implicado, los cuales se resumen en los siguientes aspectos que se presentan a continuación:

1. Mantenimiento y actualización del blog y de la página web de la SAME de la Universidad de Valencia (http://proyectosame.blogs.uv.es/comisiones/) en el cual se presentan diversos documentos y materiales audiovisuales que documentan la experiencia y el proyecto de innovación.

2. Producciones elaboradas por todas y cada una de las diferentes comisiones de trabajo implicadas en el proyecto: trípticos, carteles, noticias en prensa y en medios de comunicación, noticias en la web de la Universitat de València, noticias en la página web de la SAME, documentales, etc.

3. Desarrollo de las diferentes actividades diseñadas para la celebración de la SAME en la Facultad de Filosofía y CC. de la Educación. 
- Documento gráfico y audiovisual sobre todas las actividades que se realizan el día de la celebración de la SAME en la Facultad de Filosofía y Ciencias de la Educación.

- Documento de posicionamiento elaborado por los estudiantes y leído por ellos durante el acto principal la SAME.

4. Instrumentos de evaluación para el profesorado y alumnado de la SAME (Anexo 1): mediante la elaboración e implementación de cuestionarios de satisfacción que se llevan a cabo durante el proceso y después de la finalización del proyecto de innovación. La valoración de las mismas se planificó para que fuera realizada tanto desde la perspectiva del alumnado como la del profesorado.

En relación a los resultados que se extraen en el proceso de evaluación destacamos aquí, de modo preliminar, las ventajas de facilitar el acceso a los instrumentos de evaluación (Anexo 1) vía on-line puesto que ello ha facilitado en gram medida la recopilación de información sobre una muestra muy representativa de información en la presente edición (de un número total aproximado de 500 participantes en los talleres y 250 en la jornada, se han recabado 278 y 114 respectivamente, representando el 55,6\% y 45,6\%). Igualmente, cabe destacar que la valoración de la realización de los talleres es realmente positiva, pues en todos los aspectos sobre los que se ha preguntado nos encontramos con medias que superan el 2,5 en una escala de cinco puntos, así como, claramente, las personas que han participado en la jornada "consideran las redes sociales útiles para una movilización social como la SAME".

Más abajo, a modo ilustrativo de los momentos más significativos del proyecto, mostramos una serie de imágenes extraídas del twitter institucional de la campaña de la SAME (2018):

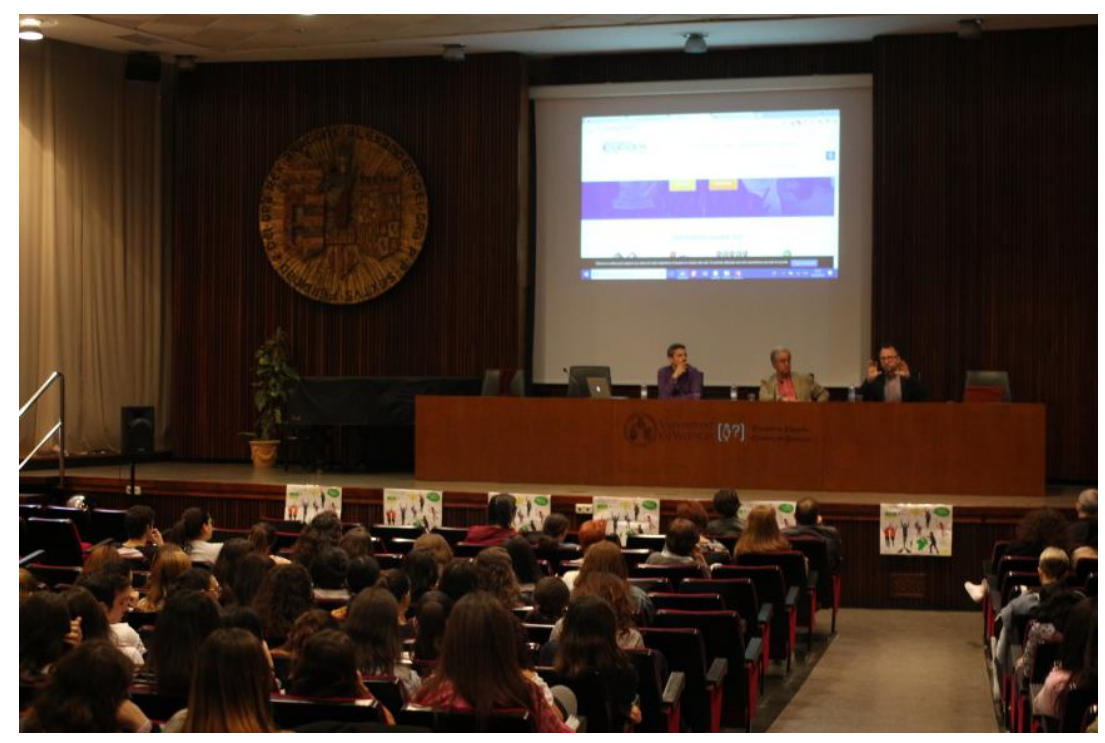

Fig. 2 Acto institucional de la SAME

(c)) EY-NC-ND 2018, Universitat Politècnica de València Congreso In-Red (2018) 


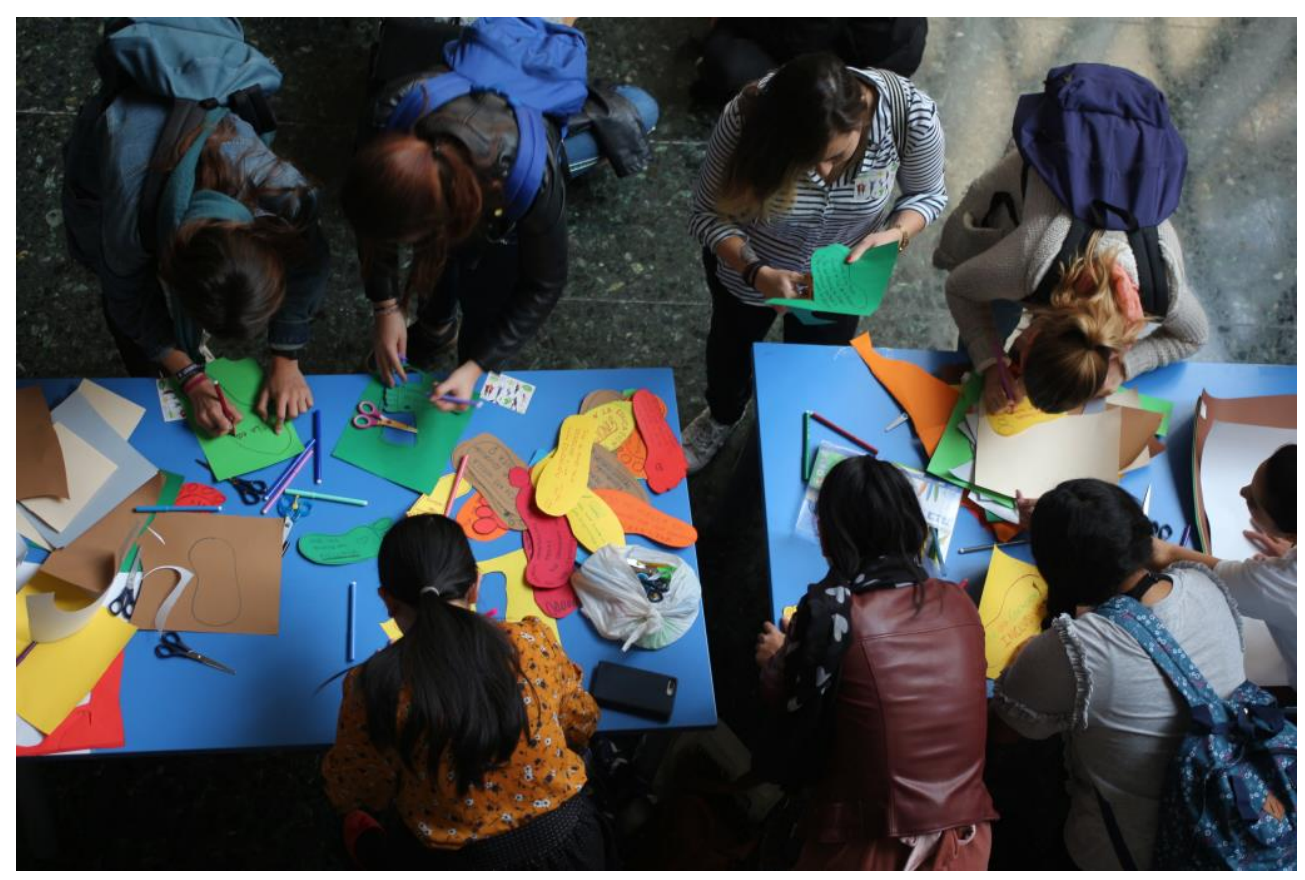

Fig. 3 Gymcana y talleres

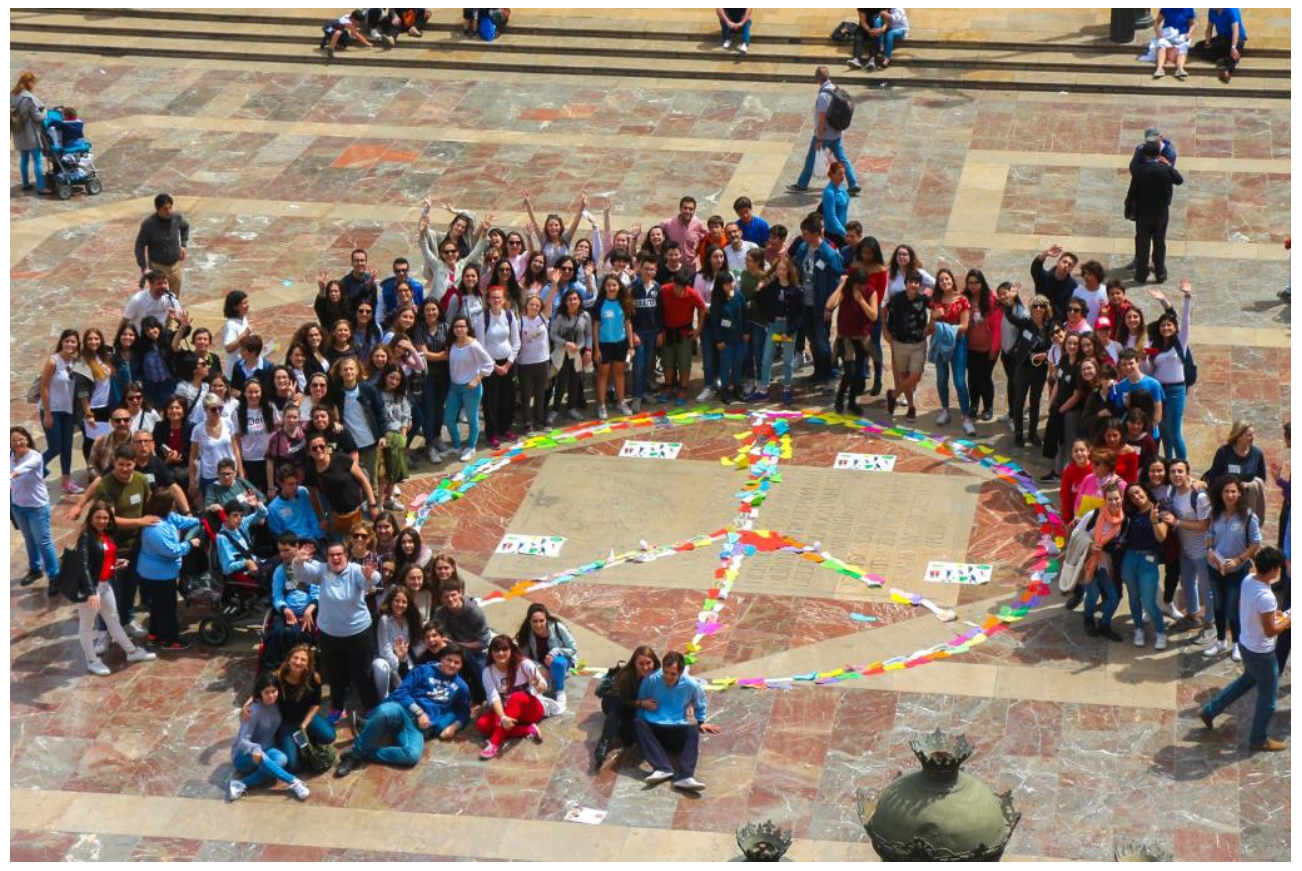

Fig. 4 Acto reivindicativo

\section{Conclusiones}

El Derecho a la Educación desgraciadamente no está al alcance de todas las personas. No obstante, desde este proyecto de innovación educativa pretendemos visualizar esta problemática y extenderla al mayor número de personas posibles para tener más 
fuerza a la hora de exigir a los representantes la erradicación de este problema y su mayor compromiso institucional para ello. Dicha realidad es una oportunidad y una obligación desde la visión de la Responsabilidad Social Universitaria, siendo conscientes de que una institución que promociona valores democráticos, unitarios, cooperativos y desde la visión del desarrollo sostenible, tiene que trabajar de manera conjunta para dotar de formación transversal en valores y compromiso ético a los agentes participantes de estas iniciativas.

Siguiendo el estudio realizado por Martínez-Usarralde, Lloret-Català y Mas-Gil (2017), a un grupo de 206 alumnos/as del grado de Educación Social de la Facultad de Filosofía y Ciencias de la Educación de la Universidad de Valencia, determinaron que estos perciben que la institución si proporciona actividades que les dan oportunidades para trabajar la dimensión ética, social y en principios que fomentan el desarrollo sostenible. Cómo críticas estos apuntaban que la coordinación y la transversalidad podría ser mejorada. Por ello, pensamos que el proyecto de la SAME por su carácter transversal, que es trabajado en el ámbito del grupo-aula en el caso de la Facultad de Filosofía y Ciencias de la Educación y mediante la semana de actividades complementarias en la Facultad de Magisterio; la participación de profesorado y ONGs en distintas comisiones, ha ido consolidando una cultura de trabajo transversal que iría en la línea de trabajo que se pretende para cumplir con los distintos objetivos, que tanto organismos internacionales, como la propia institución pretenden y desean que sean competencias basadas en la iniciativa del Campus Sostenible y del trabajo de la Responsabilidad Social Universitaria.

El éxito del proyecto de innovación que se ha consolidado en las distintas ediciones que se han celebrado desde 22013/2014 en la Universidad de Valencia, viene marcado por la coordinación de las distintas comisiones que más arriba hemos descrito. El rol de la difusión y de las nuevas tecnologías, para dar visibilidad a la campaña, ha sido fundamental para motivar la participación del alumnado de una manera activa. De hecho, este año para la celebración de la campaña se espera una gran participación a través de la red por parte del alumnado, ya que se ha erigido un concurso a través de las redes, que ya fueron un punto clave en el proyecto SAME 2016/2017. Otra de las comisiones que han sido constante en las diferentes ediciones y que ha mostrado grandes resultados en el trabajo transversal y de concienciación con el alumnado es la de la elaboración de un manifiesto crítico que se difunde en la Plaza de la Virgen de la ciudad de Valencia, esto da visibilidad al trabajo realizado en los talleres y el día de celebración de la SAME, como acto de Responsabilidad Social Universitaria a la que se le percibe un impacto fuera de las fronteras de la institución. 
Estas actividades y trabajos desde las comisiones, pensamos que son éxito y constante en las distintas ediciones por su operatividad y sentido constructivo. La diferencia viene dada por el tipo de gymkana, el foco de la campaña que modifica los talleres y las actividades que decidamos emprender con las nuevas tecnologías. Sin embargo, la conclusión más general y que dota de mayor sentido a este proyecto de innovación, es la consolidación de un grupo de trabajo y una cultura basada en la transversalidad y en la participación real que viene fortalecida por la satisfacción del alumnado con el proyecto reflejada en las distintas evaluaciones que se han realizado al término de las diferentes ediciones.

\section{Referencias}

ALVENTOSA, M. E., SENENT, J.M. y VIANA, M. I. (2018). "La formación de docentes de Primaria en las Universidades Públicas españolas. Análisis comparado de sus diseños curriculares en aspectos sociales". En II Conferência Internacional de Educação Comparada. O Professor do Século XXI em Perspectiva Comparada: Transformações e Desafios para a construção de sociedades sustentáveis. Funchal, Região Autónoma da Madeira, Portugal. P.31

BARRÓN, Á; NAVARRETE, A y FERRER-BALAS, D. (2010). "Sostenibilización Curricular En Las Universidades Españolas. ¿Ha Llegado La Hora De Actuar?" Revista Eureka sobre Enseñanza y Divulgación de las Ciencias, vol. 7, 2010, pp. 388-399. Disponible en: http://www.redalyc.org/articulo.oa?id=92013009018 (Consultada: 23 de marzo de 2018)

Bokova, I. (2014). Mensaje en video de la Sra. Irina Bokova. Directora General de la UNESCO, con motivo de la Semana de Acción Mundial 2014 sobre Educación para Todos. UNESCO. Disponible en: $\quad$ http://www.unesco.org/new/fileadmin/MULTIMEDIA/HQ/ED/pdf/GAW2014DGvideomessage-ES.pdf

CME (2018). Campaña Mundial por la Educación. CME. Disponible en: http://www.campaignforeducation.org/es/

Comunidad Valenciana. Ley 2/2003 de enero, de Consejos Sociales de las Universidades Públicas Valencianas. DOCV, 31 de enero de 2003, núm. 4430, ref. 0422/2003. Recuperado de: http://www.dogv.gva.es/disposicio-consolidadasignatura=0422/2003\&idioma=va\&L=2\&url_lista

GOMÀ, R. (2008). "La acción comunitaria: transformación social y construcción de ciudadanía". RES (Revista de Educación Social), 7. Disponible en: http://www.eduso.net/res/?b=10\&c=90\&n=251 (Consultada: 20 de marzo de 2018)

LLENA, A. y ÚCAR, X. "Acción comunitaria: miradas y diálogos interdisciplinares" en ÚCAR, X y LLENA, A. (Coords) Miradas y diálogos en torno a la acción comunitaria. Barcelona: Graó.. ISNB 10: 84-7827-447-2. ISBN 13: 978-84- 7827-447-5). Disponible en: https://www.researchgate.net/publication/282122371_Accion_comunitaria_miradas_y_dialogos_i nterdisciplinares_e_interprofesionales

MARTÍNEZ-USARRALDE, M. J.; LLORET-CATALÀ, C., y MAS-GIL, S. (2017). "Responsabilidad Social Universitaria (RSU): Principios para una Universidad Sostenible, Cooperativa y Democrática desde el Diagnóstico Participativo de su Alumnado" Archivos Analíticos de Políticas Educativas, vol. 25, 2017, pp. 1-25. Disponible en: https://epaa.asu.edu/ojs/article/view/2769/1938 (Consultada: 23 de marzo de 2018) 
SAME Valencia 2016 (@Same_UV2016).“\#Paraguada \#SAME2016 \#Valencia Gracias a tod@s por reivindicar una mayor financiación @UV_EG”.27/04/2016, 17:46, [Twitter]. https://twitter.com/SAME_UV2016 [23 de marzo de 2018:].

UNESCO (2016). Declaración de Incheon y Marco de Acción ODS 4 - Educación 2030. UNESCO. Disponible en: http://unesdoc.unesco.org/images/0024/002456/245656s.pdf

Universidad de Valencia. UV. 2016. Plà Estratègic 2016-2019. Univeritat de València. Disponible en: https://www.uv.es/uvweb/universitat/ca/pla-estrategic//pla-estrategic-2016-2019/documents-plaestrategic-2016-2019-1285972477391.html (Consultada: 23 de marzo de 2018)

Universidad de Valencia. UV. (2017). Innovación educativa. UV. Disponible en: https://www.uv.es/uvweb/servicio-formacion-permanente-innovacion-educativa/es/innovacioneducativa/convocatorias-innovacion/vigente-1285869934336.html

ANEXO 1: Instrumentos de evaluación.

\begin{tabular}{|l|}
\hline Cuestionario elaborado para la evaluación \\
\hline TALLER \\
\hline 1.- He entendido el sentido de la Semana de Acción Mundial por la Educación (SAME). \\
\hline $\begin{array}{l}\text { 2.- Con la realización del taller me he sentido motivado/a a reflexionar sobre la huella que dejamos } \\
\text { con la educación. }\end{array}$ \\
\hline $\begin{array}{l}\text { 3.- Este tipo de actividad ha sido útil para reconocer que la educación mejora a la persona y a la } \\
\text { sociedad. }\end{array}$ \\
\hline $\begin{array}{l}\text { 4.- Este tipo de actividad ha sido útil para incrementar mi actitud proactiva hacia situaciones y } \\
\text { personas que sufren la vulneración de sus derechos humanos. }\end{array}$ \\
\hline \begin{tabular}{l} 
5.- Tras la realización del Taller, tengo ganas de participar en la jornada del día 25 de abril. \\
\hline JORNADA
\end{tabular} \\
\hline 1.- Me ha gustado participar en la Semana de Acción Mundial por la Educación (SAME). \\
\hline 2.- El contenido tratado en la SAME ha sido muy interesante. \\
\hline 3.- La SAME me ha aportado una visión de cómo "la educación es el camino hacia la paz". \\
\hline 4.- La difusión de esta Jornada ha sido adecuada. \\
\hline 5.- La distribución de los diferentes actos ha sido adecuada. \\
\hline 6.- Considero las redes sociales útiles para una movilización social como la SAME. \\
\hline 7.- Volvería a participar en sucesivas ediciones en una Jornada como esta. \\
\hline
\end{tabular}

